Advances in Dynamical Systems and Applications.

ISSN 0973-5321, Volume 16, Number 1, (2021) pp. 33-43

(C) Research India Publications

https://dx.doi.org/10.37622/ADSA/16.1.2021.35-44

\title{
Bounds of the Mertens Functions
}

\author{
Darrell Cox ${ }^{a}$, Sourangshu Ghosh ${ }^{b}$, Eldar Sultanow ${ }^{c}$ \\ ${ }^{a}$ Grayson County College, Denison, TX 75020, USA \\ ${ }^{b}$ Department of Civil Engineering, Indian Institute of Technology Kharagpur, \\ West Bengal, India
}

${ }^{c}$ Potsdam University, 14482 Potsdam, Germany

\begin{abstract}
In this paper we derive new properties of Mertens function and discuss about a likely upper bound of the absolute value of the Mertens function $\sqrt{\log (x !)}>$ $|M(x)|$ when $x>1$. Using this likely bound we show that we have a sufficient condition to prove the Riemann Hypothesis.
\end{abstract}

\section{INTRODUCTION}

We define the Mobius Function $\mu(k)$. Depending on the factorization of $\mathrm{n}$ into prime factors the function can take various values in $\{-1,0,1\}$

- $\mu(n)=1$ if $\mathrm{n}$ has even number of prime factors and it is also squarefree(divisible by no perfect square other than 1)

- $\mu(n)=-1$ if $\mathrm{n}$ has odd number of prime factors and it is also square-free

- $\mu(n)=0$ if $n$ is divisible by a perfect square.

Mertens function is defined as $M(n)=\sum_{k=1}^{n} \mu(k)$ where $\mu(k)$ is the Mobius function. It can be restated as the difference in the number of square-free integers up to $x$ that have even number of prime factors and the number of square-free integers up to $x$ that have odd number of prime factors. The Mertens function rather grows very slowly since the Mobius function takes only the value $0, \pm 1$ in both the positive and 
negative directions and keeps oscillating in a chaotic manner. Mertens after verifying all the numbers up to 10,000 conjectured that the absolute value of $M(x)$ is always bounded by $\sqrt{x}$. This conjecture was later disproved by Odlyzko and te Riele ${ }^{1}$. This conjecture is replaced by a weaker one by Stieltjes ${ }^{2}$ who conjectured that $M(x)=$ $O\left(x^{\frac{1}{2}}\right)$. Littlewood ${ }^{3}$ proved that the Riemann hypothesis is equivalent to the statement that for every $\epsilon>0$ the function $M(x) x^{-\frac{1}{2}-\epsilon}$ approaches zero as $\mathrm{x} \rightarrow \infty$. This proves that the Riemann Hypothesis is equivalent to conjecture that $M(x)=O\left(x^{\frac{1}{2}+\epsilon}\right)$ which gives a rather very strong upper bound to the growth of $M(x)$. Although there exists no analytic formula, Titchmarsh ${ }^{4}$ showed that if the Riemann Hypothesis is true and if there exist no multiple non-trivial Riemann zeta function zeros, then there must exist a sequence $T_{k}$ which satisfies $k \leq T_{k} \leq k+1$ such that the following result holds:

$$
M_{0}(x)=\lim _{k \rightarrow \infty} \sum_{\substack{\rho \\|\gamma|<T_{k}}} \frac{x^{\rho}}{\rho \zeta^{\prime}(\rho)}-2+\sum_{n=1}^{\infty} \frac{(-1)^{n+1}}{(2 n) ! n \zeta(2 n+1)}\left(\frac{2 \pi}{x}\right)^{2 n}
$$

Where $\zeta(\mathrm{z})$ is the Riemann zeta function, and $\rho$ are all the all nontrivial zeros of the Riemann zeta function and $M_{0}(x)$ is defined as $M_{0}(x)=M(x)-\frac{1}{2} \mu(x)$ if $x \in Z^{+}$, $M(x)$ Otherwise (Odlyzko and te Riele ${ }^{1}$ )

\section{NEW PROPERTIES OF MERTENS FUNCTION}

Lehman ${ }^{5}$ proved that $\sum_{i=1}^{x} M(\lfloor x / i\rfloor)=1$. In general, $\sum_{i=1}^{x} M(\lfloor x /(i n)\rfloor)=1, n=$ $1,2,3, \ldots, x\left(\right.$ since $\lfloor\lfloor x / n\rfloor / i\rfloor=(\lfloor x /($ in $)\rfloor)$. Let $R^{\prime}$ denote a square matrix where element (i, j) equals 1 if $j$ divides $i$ or 0 otherwise. (In a Redheffer matrix, element $(i, j)$ equals 1 if $i$ divides $j$ or if $\mathrm{j}=1$. Redheffer ${ }^{6}$ proved that the determinant of the matrix equals the Mertens Function $M(x)$.) Let $T$ denote the matrix obtained from $R^{\prime}$ by element-by-element multiplication of the columns by $M\left(\left\lfloor\frac{x}{1}\right\rfloor\right), M\left(\left\lfloor\frac{x}{2}\right\rfloor\right), M\left(\left\lfloor\frac{x}{3}\right\rfloor\right) \ldots M\left(\left\lfloor\frac{x}{x}\right\rfloor\right)$. For example, the T matrix for $\mathrm{x}=12$ is

$$
T=\left[\begin{array}{cccccccccccc}
-2 & 0 & 0 & 0 & 0 & 0 & 0 & 0 & 0 & 0 & 0 & 0 \\
-1 & -1 & 0 & 0 & 0 & 0 & 0 & 0 & 0 & 0 & 0 & 0 \\
-1 & 0 & -1 & 0 & 0 & 0 & 0 & 0 & 0 & 0 & 0 & 0 \\
-1 & -1 & 0 & -1 & 0 & 0 & 0 & 0 & 0 & 0 & 0 & 0 \\
0 & 0 & 0 & 0 & 0 & 0 & 0 & 0 & 0 & 0 & 0 & 0 \\
0 & 0 & 0 & 0 & 0 & 0 & 0 & 0 & 0 & 0 & 0 & 0 \\
1 & 0 & 0 & 0 & 0 & 0 & 1 & 0 & 0 & 0 & 0 & 0 \\
1 & 1 & 0 & 1 & 0 & 0 & 0 & 1 & 0 & 0 & 0 & 0 \\
1 & 0 & 1 & 0 & 0 & 0 & 0 & 0 & 1 & 0 & 0 & 0 \\
1 & 1 & 0 & 0 & 1 & 0 & 0 & 0 & 0 & 1 & 0 & 0 \\
1 & 0 & 0 & 0 & 0 & 0 & 0 & 0 & 0 & 0 & 1 & 0 \\
1 & 1 & 1 & 1 & 0 & 1 & 0 & 0 & 0 & 0 & 0 & 1
\end{array}\right]
$$


Theorem (1) : $\sum_{i=1}^{x} M(\lfloor x / i\rfloor) i=A(x)$

Proof: Let us now take $A(x)=\sum_{i=1}^{x} \varphi(\mathrm{i})$ where $\varphi$ is Euler's totient function. Let $U$ denote the matrix obtained from $T$ by element-by-element multiplication of the columns by $\varphi(j)$. The sum of the columns of $U$ then equals $A(x)$. Now since $i=$ $\sum_{d \mid i} \varphi(\mathrm{d})$ we can write $\sum_{i=1}^{x} M(\lfloor x / i\rfloor) i$ (the sum of the rows of $\mathrm{U}$ ) equals $A(x)$.

By the Schwarz inequality, $A(x) / \sqrt{x(x+1)(2 x+1) / 6}$ is a lower bound of $\sqrt{\sum_{i=1}^{x} M(\lfloor x / i\rfloor)^{2}} \cdot A(x)=\sum_{i=1}^{x} \varphi(\mathrm{i})$ Is further simplified by Walfisz ${ }^{7}$ and Hardy and Wright ${ }^{8}$ as

$$
\begin{gathered}
A(x)=\sum_{i=1}^{x} \varphi(\mathrm{i})=\frac{1}{2} \sum_{k=1}^{x} \mu(k)\left\lfloor\frac{x}{k}\right\rfloor\left(1+\left\lfloor\frac{x}{k}\right\rfloor\right)=\frac{3}{\pi^{2}} x^{2}+ \\
O\left(n(\log n)^{2 / 3}(\log \log n)^{4 / 3}\right) \\
\sqrt{\sum_{i=1}^{x} M(\lfloor x / i\rfloor)^{2}}>\frac{A(x)}{\sqrt{\frac{x(x+1)(2 x+1)}{6}}}>\frac{\frac{3}{\pi^{2}} x^{2}}{\sqrt{\frac{x(x+1)(2 x+1)}{6}}}=\frac{3 \sqrt{3}}{\pi} \frac{x^{2}}{\sqrt{x(x+1)(x+1 / 2)}}
\end{gathered}
$$

This can be further simplified to

$$
\sqrt{\left.\sum_{i=1}^{x} M(\mid x / i]\right)^{2} / x}>\frac{3 \sqrt{3}}{\pi} \frac{1}{\sqrt{(1+1 / x)(1+1 / 2 x)}}
$$

Taking limit of infinity on both the sides, we get

$$
\lim _{x \rightarrow \infty} \sqrt{\sum_{i=1}^{x} M(\lfloor x / i\rfloor)^{2} / x}>\frac{3 \sqrt{3}}{\pi}
$$

This shows that $\sum_{i=1}^{x} M(\lfloor x / i\rfloor)^{2}$ at large values of $\mathrm{x}$ is greater than $\frac{27}{\pi^{2}} x^{2}$.

Let $\Lambda(i)$ denote the Mangoldt function $\left(\Lambda(i)\right.$ equals $\log (p)$ if $i=p^{m}$ for some prime $p$ and some $m \geq 1$ or 0 otherwise). Mertens ${ }^{9}$ proved that $\sum_{i=1}^{x} M(\lfloor x / i\rfloor) \log i$ $=\psi(x)$ where $\psi(x)$ denotes the second Chebyshev function $\left(\psi(\mathrm{x})=\sum_{i \leq x} \Lambda(i)\right)$.

Theorem (2) : $\sum_{i=1}^{x} M\left(\left\lfloor\frac{x}{i}\right\rfloor\right) \log (i) \sigma_{0}(i) / 2=\log (x !)$

Proof: Let $\sigma_{x}(i)$ denote the sum of positive divisors function $\left(\sigma_{x}(i)=\sum_{d \mid i} d^{x}\right)$. Replacing $\varphi(j)$ with $\log (j)$ in the $\mathrm{U}$ matrix gives a similar result.

Let $\lambda(n)$ denote the Liouville function $\left(\lambda(1)=1\right.$ or if $=p_{1}^{a_{1}} . . p_{k}^{a_{k}}, \lambda(n)=$ $\left.(-1)^{a_{1}+. .+a_{k}}\right) . \sum_{d \mid n} \lambda(d)$ Equals 1 if $\mathrm{n}$ is a perfect square or 0 otherwise. Let $(x)=$ $\sum_{\mathrm{n} \leq \mathrm{x}} \lambda(d)$. Let us also assume $H(x)=\sum_{\mathrm{n} \leq \mathrm{x}} \mu(n) \log (n) . H(x) /(x \log (x)) \rightarrow 0$ as $x \rightarrow \infty$ and $\left.\lim _{x \rightarrow \infty} M(x) / x-H(x) /(x \log (x))\right)=0$. The statement $\lim _{x \rightarrow \infty} M(x) /$ $x=0$ is equivalent to the prime number theorem. Also, $\Lambda(n)=-\sum_{\mathrm{d} \mid \mathrm{n}} \mu(d) \log (d)$. $\left(\right.$ Apostol $\left.{ }^{10}\right)$.

The generalization of the Euler's totient function is Jordan totient function. Let it be denoted as $J_{k}(x)$ which is defined as number of set of $\mathrm{k}$ positive integers which are all less than or equal to $\mathrm{n}$ that will form a co-prime set of $(k+1)$ positive integers 
together with $n$. Let us define $(x)=\sum_{i=1}^{x} J_{k}(i)$. It is known that $\sum_{d \mid n} J_{k}(d)=n^{k}$. Then we get the following theorem.

Theorem (3): $\sum_{i=1}^{x} M\left(\left\lfloor\frac{x}{i}\right\rfloor\right) i^{k}=B(x)$ $B(x)$ is expanded by McCarthy ${ }^{11}$ to be

$$
B(x)=\sum_{i=1}^{x} J_{k}(i)=\frac{n^{r+1}}{(r+1) \zeta(\mathrm{r}+1)}+O\left(n^{r}\right)
$$

We therefore get $\sum_{i=1}^{x} M\left(\left\lfloor\frac{x}{i}\right\rfloor\right) i^{k}=B(x)=\frac{n^{k+1}}{(k+1) \zeta(\mathrm{k}+1)}+O\left(n^{k}\right)$.

Likewise we can derive some other similar relationships using the $T$ matrix that are as listed below:

Theorem (4): $\sum_{i=1}^{x} M\left(\left\lfloor\frac{x}{i}\right\rfloor\right) \sigma_{k}(i)=\sum_{i=1}^{x} i^{k} \quad$ for $k \in Z^{+}$

Theorem (5): $\sum_{i=1}^{x} M\left(\left\lfloor\frac{x}{i}\right\rfloor\right)$ where the summation is over those $i$ values that are perfect squares equals $L(x)$

Theorem (6): $\sum_{i=1}^{x} M\left(\left\lfloor\frac{x}{i}\right\rfloor\right) \Lambda(i)=-H(x)$

Theorem (7): $\sum_{i=1}^{x} M\left(\left\lfloor\frac{x}{i}\right\rfloor\right) 2^{\omega(n)}=\sum_{i=1}^{x}|\mu(i)| \sim \frac{6}{\pi^{2}} x^{2}+O(\sqrt{n})=$ No. of Square Free Integers

Theorem (8): $\sum_{i=1}^{x} M\left(\left\lfloor\frac{x}{i}\right\rfloor\right) d\left(n^{2}\right)=\sum_{i=1}^{x} 2^{\omega(i)} \quad$ where $d(x)$ is the sum of all the divisors of $x$

Theorem (9): $\sum_{i=1}^{x} M\left(\left\lfloor\frac{x}{i}\right\rfloor\right) d^{2}(n)=\sum_{i=1}^{x} d\left(i^{2}\right)$

Theorem (10): $\sum_{i=1}^{x} M\left(\left\lfloor\frac{x}{i}\right\rfloor\right)\left(\frac{i}{\varphi(\mathrm{i})}\right)=\sum_{i=1}^{x} \frac{\mu^{2}(i)}{\varphi(\mathrm{i})}$

Similarly many other relationships can be found between various arithmetic functions and the Mertens Functions.

\section{A LIKELY UPPER BOUND OF $|\mathrm{M}(\mathbf{x})|$}

The following conjecture is based on data collected for $\mathrm{x} \leq 500,000$.

Conjecture (1): $\log (x !)>\sum_{i=1}^{x} M(\lfloor x / i\rfloor)^{2}>\psi(x)$ when $x>7$

By Stirling's formula, $\log (x !)=x \log (x)-x+O(\log (x))$, since $\log (x)$ increases more slowly than any positive power of $\log (x)$, this is a better upper bound of $\sum_{i=1}^{x} M(\lfloor x / i\rfloor)^{2}$ than $x^{1+\varepsilon}$ for any $\varepsilon>0$. This likely bound can be used to prove the Riemann Hypothesis since $\sum_{i=1}^{x} M(\lfloor x / i\rfloor)>|M(x)|$ and therefore we can write $\sqrt{\log (x !)}>|M(x)|$. Since the growth of $\left.\sum_{i=1}^{x} M(\mid x / i]\right)^{2}$ is lesser than $x^{1+\varepsilon}$ for any $\varepsilon>0$. We can say

$$
M(x) x^{-\frac{1}{2}-\epsilon} \rightarrow 0 \text { as } x \rightarrow \infty
$$


Figure 1 for a plot of $(x !), \sum_{i=1}^{x} M(\lfloor x / i\rfloor)^{2}$, and $\psi(x)$ for $x=1,2,3, \ldots, 1000$.

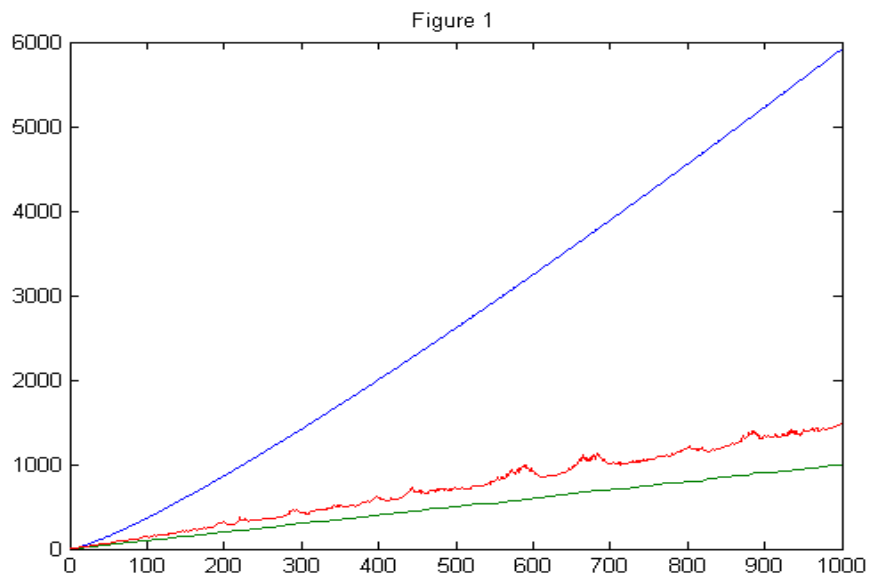

Fig 1: Plot of $\log (x !), \sum_{i=1}^{x} M(\lfloor x / i\rfloor)^{2}$, and $\psi(x)$ for $x=1,2,3, \ldots, 1000$

Let $j(x)=\sum_{i=1}^{x} M(\lfloor x / i\rfloor)^{2}$ where the summation is over $i$ values where $i \mid x$. Let $l_{1}, l_{2}, l_{3}$ denote the $\mathrm{x}$ values where $j(x)$ is a local maximum (that is, greater than all preceding $j(x)$ values) and let $m_{1}, m_{2}, m_{3} \ldots$. denote the values of the local maxima. The local maxima occur at $\mathrm{x}$ values that equal products of powers of small primes (Lagarias $^{12}$ discussed colossally abundant numbers and their relationship to the Riemann hypothesis). See Figure 2 for a plot of $l_{i} /\left(\log \left(l_{i}\right) m_{i}\right), m_{i} / l_{i}$, and $1 / \log \left(l_{i}\right)$ for $i=1,2,3, \ldots, 772$ (corresponding to the local maxima for $\mathrm{x} \leq 15,000$, $000,000) .\left(M(x)\right.$ Values for large $\mathrm{x}$ were computed using Del'eglise and Rivat's ${ }^{13}$ algorithm.) The first two curves cross frequently, so there are $i$ values where mi is approximately equal to $l_{i} / \sqrt{\log \left(l_{i}\right)}$.

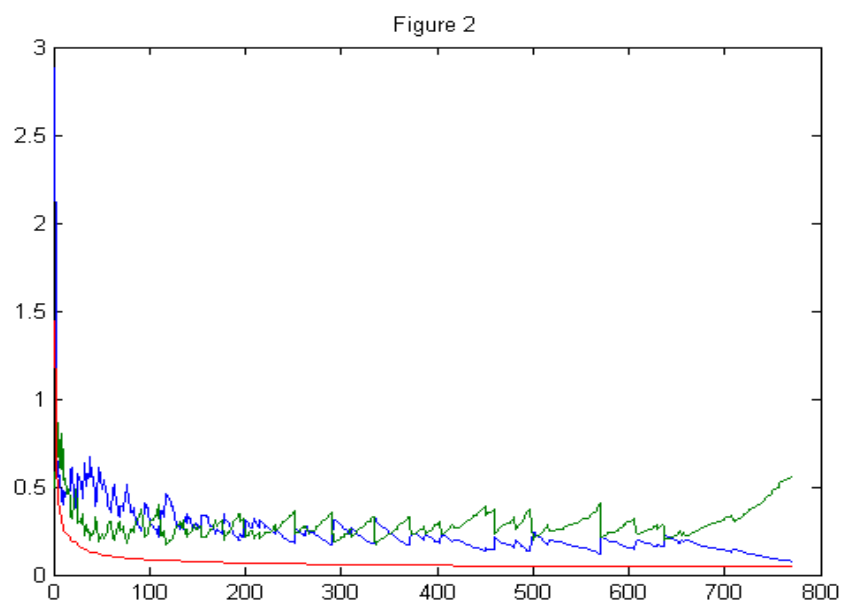

Fig 2: Plot of $l_{i} /\left(\log \left(l_{i}\right) m_{i}\right), m_{i} / l_{i}$, and $1 / \log \left(l_{i}\right)$ for $i=1,2,3, \ldots, 772$ 
See Figure 3 for a plot of $j(x)$ and $\sum_{i=1}^{x} M(\lfloor x / i\rfloor)^{2}$ for $\mathrm{x}=1,2,3, \ldots, 10,000$. See Figure 4 for a plot of $\log \left(l_{i}\right), \log \left(m_{i}\right), \log \left(M\left(l_{i}\right)^{2}\right)$, and $\log \left(m_{i} / \sigma_{0}\left(l_{i}\right)\right)$ for $\mathrm{i}=1,2$, $3, \ldots, 772$ (when $M(l i)=0, \log (M(l i) 2$ ) is set to -1 ). See Figure 5 for a plot of $\left|M\left(l_{i}\right)\right| / \sqrt{ } l_{i}$ for $i=1,2,3, \ldots, 772$. The largest known value of $|M(x)| / \sqrt{ } x$ (computed by Kotnik and van de Lune ${ }^{14}$ for $\mathrm{x} \leq 1014$ ) is 0.570591 (for $M(7,766,842,813)=50,286)$. The largest $\left|M\left(l_{i}\right)\right| / \sqrt{ } l_{i}$ value for $\mathrm{x} \leq 15,000$, 000,000 is 0.568887 (for $l_{i}=7,766,892,000$ ). The largest known value of $|M(x)| /$ $\sqrt{x} \quad$ (computed by Kuznetsov ${ }^{15}$ is 0.585767684 (for $M(11,609,864,264,058,592,345)=-1,995,900,927)$.

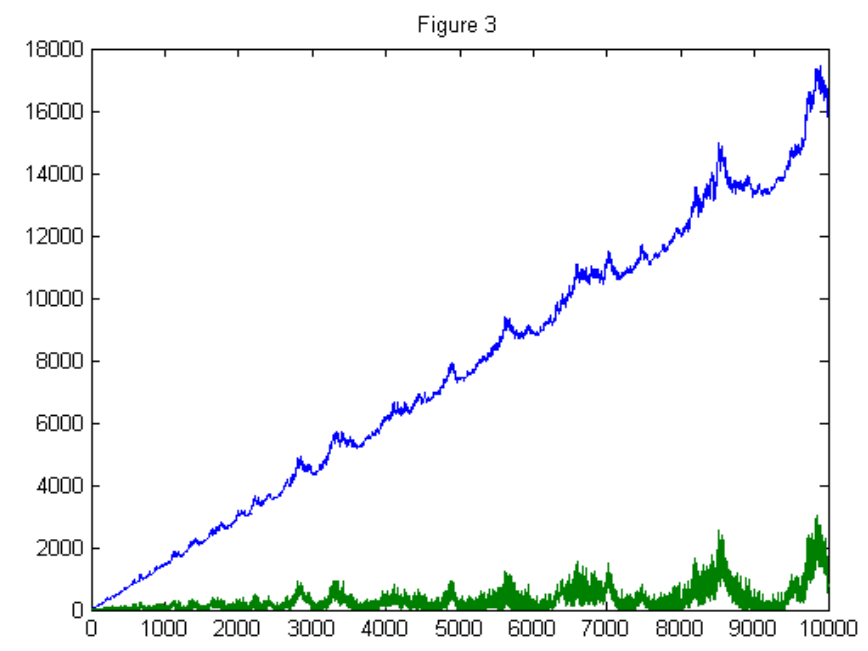

Fig 3: Plot of $j(x)$ and $\sum_{i=1}^{x} M(\lfloor x / i\rfloor)^{2}$ for $\mathrm{x}=1,2,3, \ldots, 10,000$

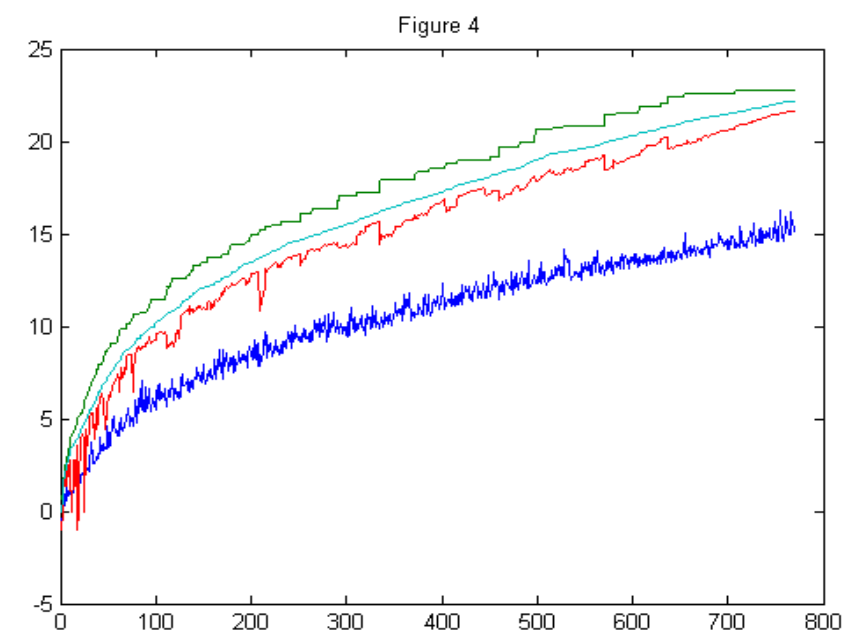

Fig 4: Plot of $\log \left(l_{i}\right), \log \left(m_{i}\right), \log \left(M\left(l_{i}\right)^{2}\right)$, and $\log \left(m_{i} / \sigma_{0}\left(l_{i}\right)\right)$ for $\mathrm{i}=1,2,3, \ldots, 772$ 


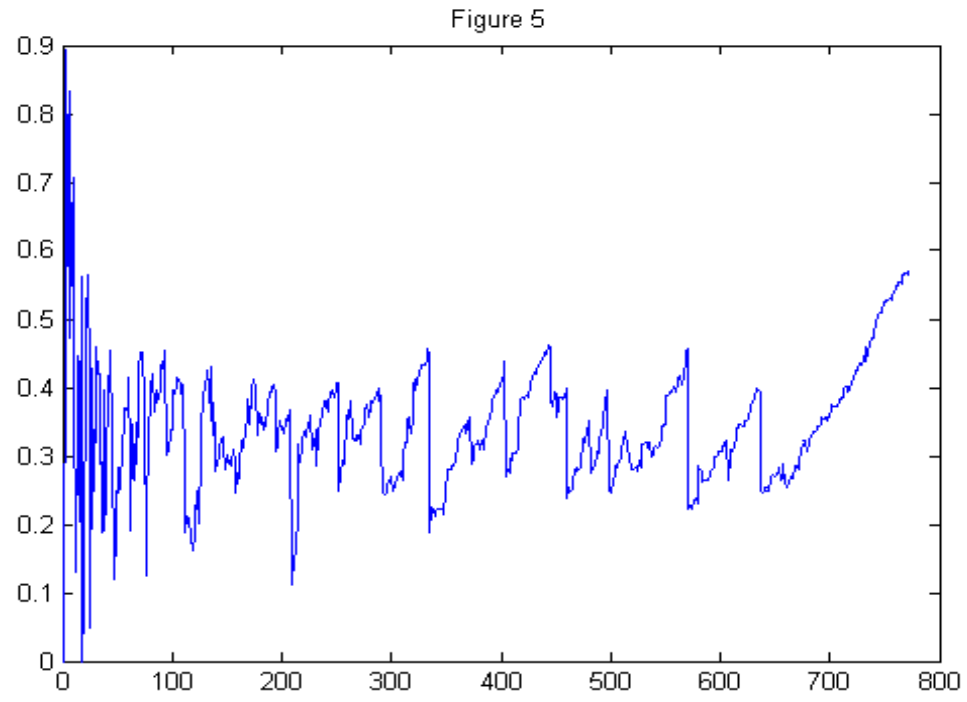

Fig 5: Plot of $\left|M\left(l_{i}\right)\right| / \sqrt{l_{i}}$ for $i=1,2,3, \ldots, 772$

Let $l_{i}$ and $m_{i}$ be similarly defined for the function $\sigma_{0}(x) .\left(l_{i}, i=1,2,3, \ldots\right.$ are known as "highly composite" numbers. Ramanujan ${ }^{16}$ initiated the study of such numbers. Robin ${ }^{17}$ computed the first 5000 highly composite numbers.) Let $m_{i}^{\prime}$ denote $j\left(l_{i}\right)$. See Figure 6 for a plot of $l_{i} /\left(\log \left(l_{i}\right) m_{i}^{\prime}\right), m_{i}^{\prime} / l_{i}$, and $1 / \log \left(l_{i}\right)$ for $i=$ $2,3,4, \ldots, 160$ (corresponding to the local maxima for $x \leq$ $2,244,031,211,966,544,000)$. (M(x) values for large $\mathrm{x}$ were computed using an algorithm similar to that used by Kuznetsov. The computations were done on an Intel i7-6700K CPU with 64 GB of RAM.) Although the first two curves cross frequently, $m_{i}^{\prime}$ does not appear to converge to $l_{i} / \sqrt{\log \left(l_{i}\right)}$.

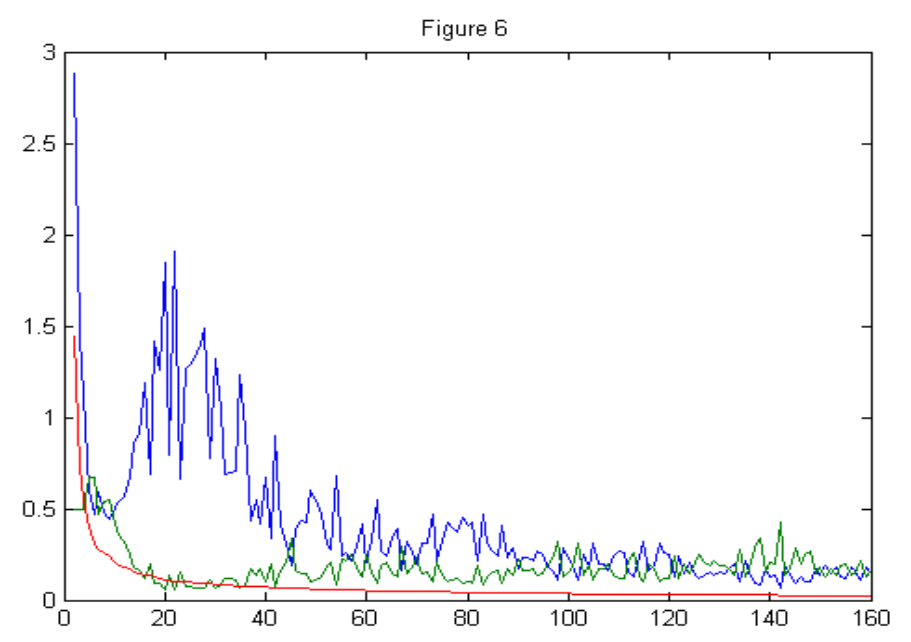

Fig 6: Plot of $l_{i} /\left(\log \left(l_{i}\right) m_{i}^{\prime}\right), m_{i}^{\prime} / l_{i}$, and $1 / \log \left(l_{i}\right)$ for $i=2,3,4, \ldots, 160$ 


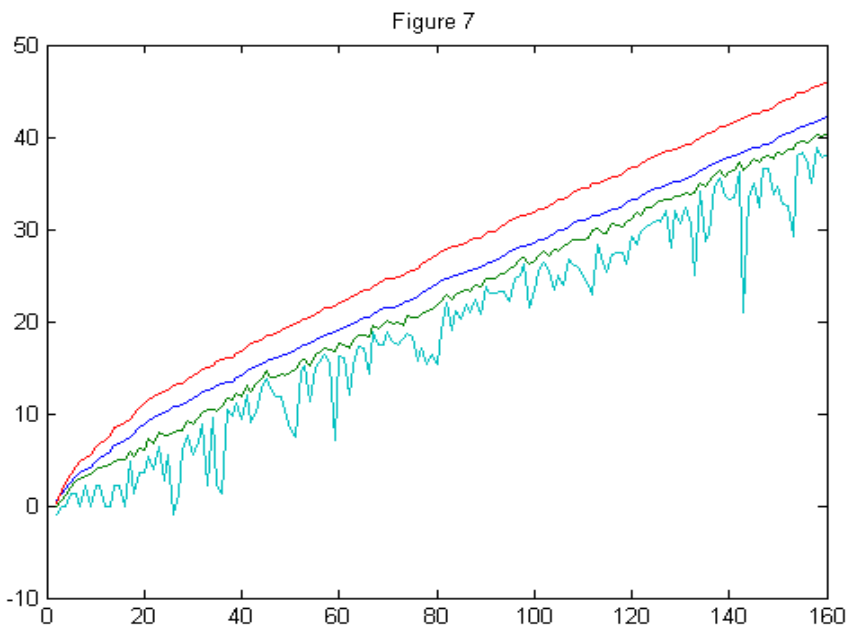

Fig 7: Plot of $\log \left(l_{i}\right)+\log \left(\log \left(l_{i}\right)\right), \log \left(l_{i}\right), \log \left(m_{i}^{\prime}\right)$, and $\log \left(M\left(l_{i}\right)^{2}\right)$, for $i=2,3,4, \ldots, 160$

See Figure 7 for a plot of $\log \left(l_{i}\right)+\log \left(\log \left(l_{i}\right)\right), \log \left(l_{i}\right), \log \left(m_{i}^{\prime}\right)$, and $\log \left(M\left(l_{i}\right)^{2}\right)$, for $i=2,3,4, \ldots, 160$ (when $M\left(l_{i}\right)=0, \log \left(M\left(l_{i}\right)^{2}\right)$, is set to $-1)$. The vertical distance between the first and third curves appears to become roughly constant. See Figure 8 for a plot of $\left(\log \left(l_{i}\right)+\log \left(\log \left(l_{i}\right)\right)-\log \left(m_{i}^{\prime}\right)\right.$,for i $=2,3,4 \ldots 160$. See Figure 9 for a plot of $\log \left(l_{i}\right)+\frac{1}{2} \log \left(\log \left(l_{i}\right)\right), \log \left(\sum_{i=1}^{l_{i}} M\left(\left[l_{i} /\right.\right.\right.$ $\left.i])^{2}\right)$, and $\log \left(l_{i}\right)$ for $i=2,3,4, \ldots, 160 . \log \left(l_{i}\right)+\frac{1}{2} \log \left(\log \left(l_{i}\right)\right)$ Is greater than $\log \left(\sum_{n=1}^{l_{i}} M\left(\left\lfloor l_{i} / n\right\rfloor\right)^{2}\right)$ and $\log \left(\sum_{n=1}^{l_{i}} M\left(\left\lfloor l_{i} / n\right\rfloor\right)^{2}\right)$ is greater than $\log \left(l_{i}\right)$ for $\mathrm{i}>4$. This is evidence in support of Conjecture 1. See Figure 10 for a plot of $\log \left(l_{i}\right)+$ $\frac{1}{2} \log \left(\log \left(l_{i}\right)\right)-\log \left(\sum_{n=1}^{l_{i}} M\left(\left\lfloor l_{i} / n\right\rfloor\right)^{2}\right)$ for $i=2,3,4, \ldots, 160$.

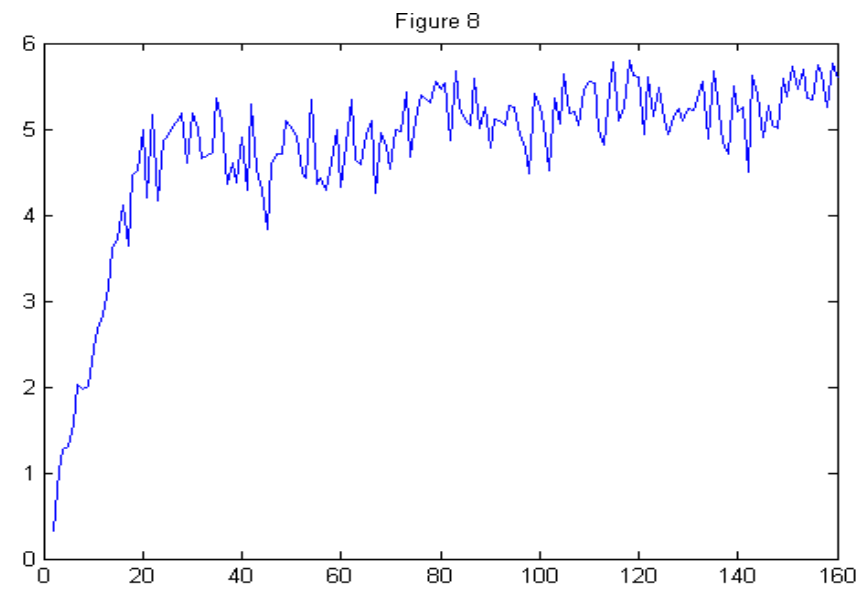

Fig: 8 for a plot of $\left(\log \left(l_{i}\right)+\log \left(\log \left(l_{i}\right)\right)-\log \left(m_{i}^{\prime}\right)\right.$,for $\mathrm{i}=2,3,4 \ldots 160$. 


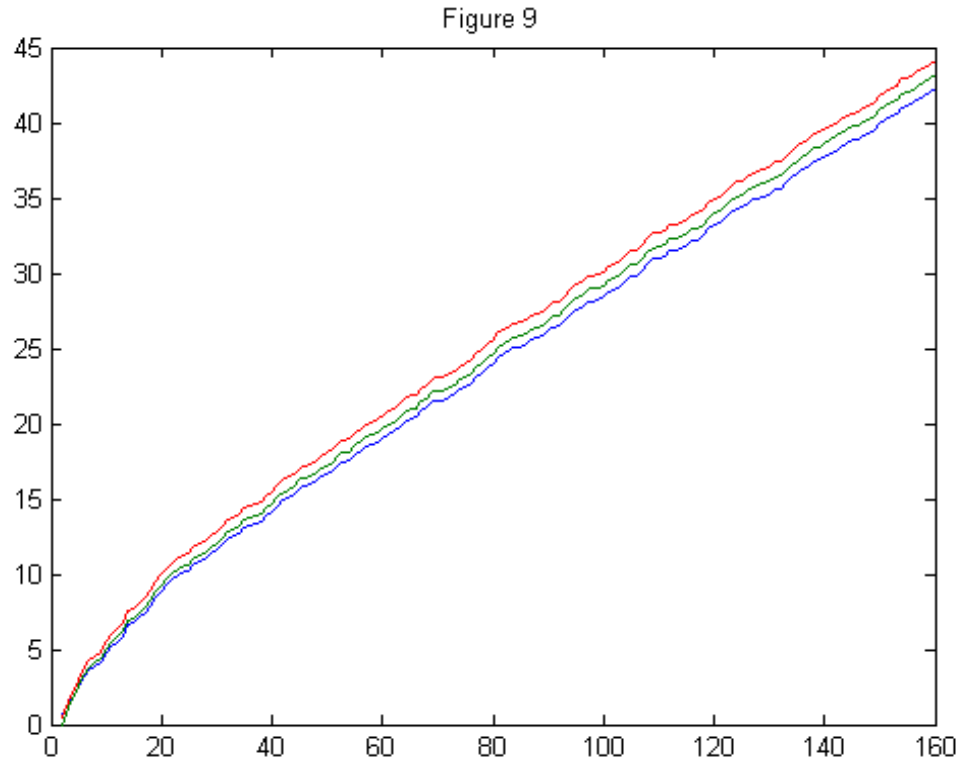

Fig 9: Plot of $\log \left(l_{i}\right)+\frac{1}{2} \log \left(\log \left(l_{i}\right)\right), \log \left(\sum_{i=1}^{l_{i}} M\left(\left\lfloor l_{i} / i\right\rfloor\right)^{2}\right)$, and $\log \left(l_{i}\right)$ for $i=2,3,4, \ldots, 160$

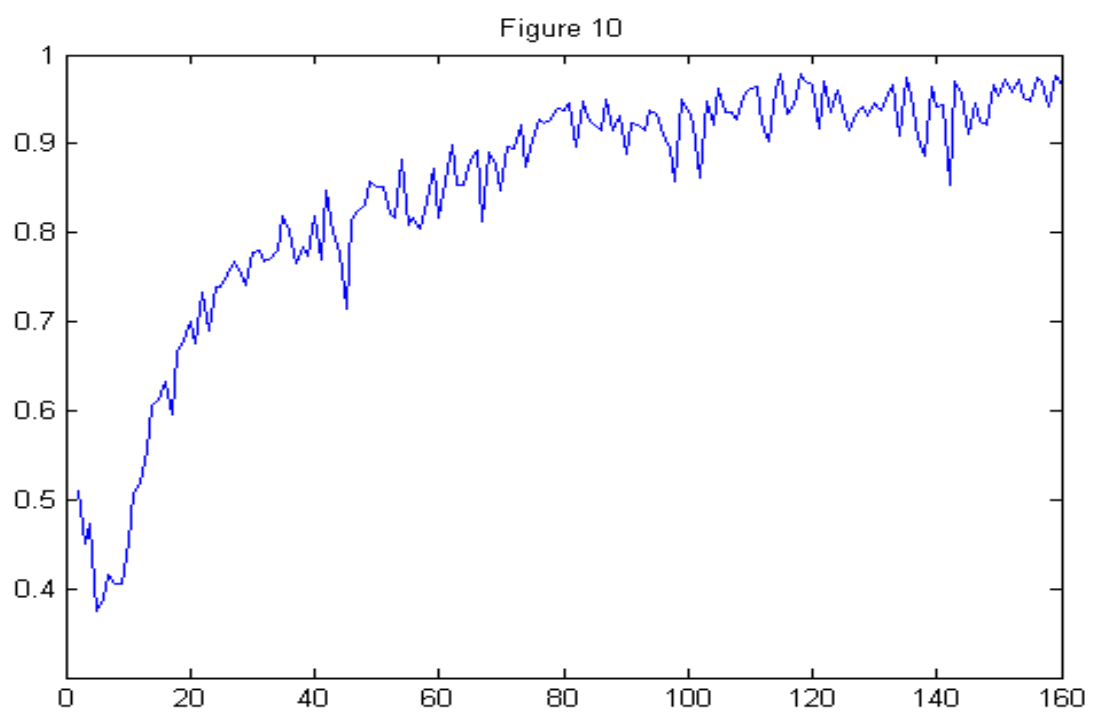

Fig 10: Plot of $\log \left(l_{i}\right)+\frac{1}{2} \log \left(\log \left(l_{i}\right)\right)-\log \left(\sum_{n=1}^{l_{i}} M\left(\left\lfloor l_{i} / n\right\rfloor\right)^{2}\right)$ for $i=2,3,4, \ldots, 160$. 


\section{CONCLUSION}

In this paper we derived new relations between Mertens function with a different arithmetic functions and also discussed about a likely upper bound of the absolute value of the Mertens function $\sqrt{\log (x !)}>|M(x)|$ when $x>1$ with sufficient numerical evidence. More experimental evidence can be found in the work of Cox and Ghosh ${ }^{18-19}$.Using this likely upper bound we showed that we have a sufficient condition to prove the Riemann Hypothesis using the Littlewood condition.

\section{REFERENCES}

[1] Odlyzko, A. M. and te Riele, H. J. J. "Disproof of the Mertens Conjecture." J. reine angew. Math. 357, 138-160, 1985.

[2] Stieltjes, T. C. R. A. S. 1885.

[3] J. E. Littlewood, Quelques cons_equences de l'hypoth_ese que la fonction_(s) n'a pas de z_eros dans le demi-plan $\operatorname{Re}(\mathrm{s})>1=2$. C. R. Acad. Sci. Paris 154, 263-266 (1912)

[4] Titchmarsh, E. C. The Theory of Functions, 2nd ed. Oxford, England: Oxford University Press, 1960.

[5] R. S. Lehman, On Liouville's Function, Math. Comput. 14:311-320 (1960)

[6] R. M. Redheffer, Eine explizit losbare Optimierungsaufgabe, Internat. Schriftenreine Numer. Math., 36 (1977)

[7] Walfisz, A. Ch. 5 in Weyl's exponential sums in modern number theory. Berlin: Deutscher Verlag der Wissenschaften, 1963.

[8] Hardy, G.H. and Wright, E.M. "The Average Order of phi (n)." \$18.5 in An Introduction to the Theory of Numbers, 5th ed. Oxford, England: Clarendon Press, pp. 268-269, 1979

[9] F. Mertens, Uber eine zahlentheoretische Funktion, Akademie Wissenschaftlicher Wien Mathematik-Naturlich Kleine Sitzungsber, 106 (1897) 761-830

[10] T. M. Apostol, Introduction to Analytic Number Theory, Springer, 1976

[11] McCarthy, P.J.: Introduction to Arithmetical Functions. Springer, New York (1986)

[12] J. C. Lagarias, An elementary problem equivalent to the Riemann hypothesis, American Mathematical Monthly 109, 534-543 (2002)

[13] M. Del_eglise and J. Rivat, Computing the Summation of the Mobius Function, Experimental Mathematics 5, 291-295 (1996)

[14] T. Kotnik and J. van de Lune, On the order of the Mertens function, Experimental Mathematics 13, pp. 473-481 (2004)

[15] E. Kuznetsov, Computing the Mertens function on a GPU, arXiv:1108.0135v1 [math.NT], (2011) 
[16] S. Ramanujan, Highly composite numbers, Proc. London Math. Soc. 14 (1915), 347-407

[17] G. Robin, M_ethodes d'optimalisation pour un probl_eme de th_eories des nombres., RAIRO Inform. Th_eor. 17, 239-247, 1983

[18] Cox, Darrell \& Ghosh, Sourangshu. (2021). Signs of the Mertens function. 10.13140/RG.2.2.26074.47042.

[19] Cox, Darrell \& Sultanow, Eldar \& Ghosh, Sourangshu. (2021). The Energy Spectral Density of the Mertens Function. 10.13140/RG.2.2.33037.67043. 
